\title{
Does Lifestyle Impact Risk, Burden, and Symptomatology of Atrial Fibrillation?
}

Ratika Parkash MD MS

DOI: 10.22374/cjgim.v13iSP1.310

\author{
About the Author \\ Ratika Parkash is with the QEII Health Sciences Center, Halifax, Nova Scotia. \\ Correspondence may be directed to: Ratika.parkash@nshealth.ca
}

Atrial fibrillation is the most common sustained arrhythmia, affecting $1-2 \%$ of the general population and $8 \%$ of patients over the age of 80 years. ${ }^{1}$ The lifetime risk for development of AF is $26 \%$ for men and $23 \%$ for women. It is associated with significant morbidity, mortality and cost, but also with an increase in mortality and a six-fold risk of stroke., ${ }^{2,3}$ The Framingham heart study showed that AF was associated with a 1.5 to 1.9 fold mortality risk after adjustment for the preexisting $\mathrm{CV}$ conditions with which AF was related. ${ }^{4}$ The Heart and Stroke Foundation estimates that 350000 Canadians are living with atrial fibrillation, and that this is increasing due to Canada's aging population. ${ }^{5}$

The diagnosis of AF may be made fortuitously, such as through a pulse check, or in the emergency department if the patients presents with symptoms. Severity of symptoms for AF may range from a 'nuisance' feeling of palpitations to debilitating symptoms that do not permit performance of activities of daily living, interfere with normal livelihood and significantly impair exercise tolerance. The most severe symptoms are those resulting in hemodynamic compromise and heart failure, which are associated with poor prognosis and increased mortality. ${ }^{6}$ Those that are symptomatic pose the greatest burden to the health care system, often making repeated visits to the emergency room for treatment or repeated hospitalizations due to delayed and limited access to specialist care, to initiate necessary therapies or undergo crucial cardiac investigations. Those that are asymptomatic don't pose as great a burden, at the time of diagnosis, however, are at risk for deleterious consequences if not treated appropriately. The mainstay for therapy for $\mathrm{AF}$ is aimed at stroke prevention and control of symptoms due to the arrhythmia itself. The primary mode of therapy is to use rate controlling agents, such as calcium channel blockers or beta blockers, but patients with more symptoms may require rhythm control. In one study, AF was diagnosed by the family physician in $63.2 \%$ of events, by an office-based cardiologist in $13.2 \%$, and by hospital-based physicians in $23.9 \%$ of cases. ${ }^{7}$ Up to a third of patients with AF will present to an emergency department (ED) at some time due to symptoms, representing 3-6\% of all medical admissions to hospital.1 Overall, the estimated care costs of hospitalized AF patients are 9 to 23-fold greater than for those without $\mathrm{AF}^{8}$

Most AF care is delivered through family physicians, eventual referral to specialists, but many repeated emergency room visits, or even hospitalizations may have occurred, prior to specialist assessment. Mcdonald et al. reported an $88 \%$ increase in $\mathrm{ED}$ visits with a primary diagnosis of AF over a 12 year period (1993 to 2004). ${ }^{9}$ Of these, $64 \%$ were admitted to hospital; this proportion remained constant over the 12 year period. There are few data on the actual costs to the health care system for AF. Khaykin et al. estimated a cost of $\$ 4840 /$ patient/year of $\mathrm{AF}^{10}$ The distribution of costs are amongst inhospital care, emergency room visits and family physician visits. There are many aspects of AF management that are best managed by those specifically trained to manage AF. It is well documented that there are a number of care gaps in management of AF at the general practitioner level. ${ }^{11,12}$ These issues relate to which patients to anticoagulate, when to perform a cardioversion, when to switch from rate to rhythm control, and when to refer for catheter ablation.

Pathophysiology of AF as it relates to cardiovascular risk factors

AF may be due to one of three mechanisms: ${ }^{13}$ the initiation and perpetuation of atrial fibrillation requires triggers which start an episode, and an arrhythmogenic substrate which allows it to continue. The triggers typically consist of ectopy which most frequently originate in the pulmonary veins, but extra-pulmonary 
vein triggers may also exist in the coronary sinus, superior vena cava or within the body of the left atrium. ${ }^{14,15}$ The maintenance of $\mathrm{AF}$, once triggered, is due to changes in atrial structural and electrophysiologic properties. These changes may result from a wide variety of causes including left atrial stretch, structural remodeling leading to atrial fibrosis, as in congestive heart failure. ${ }^{16}$ Paroxysms of rapid, consecutive premature atrial contractions, or short paroxysms of AF, can promote further functional changes that can perpetuate $\mathrm{AF}^{17}$ The role of the autonomic nervous system in the maintenance of AF has also been extensively studied. The ganglionic plexi are located along the great vessels and in the pericardial fat pads. Scherlag et al. have performed numerous experimental studies demonstrating that activation of the ganglionic plexi at the junctions of the pulmonary vein and atrium may result in conversion of pulmonary vein ectopy to atrial fibrillation. ${ }^{18}$ Kistler et al. studied the effect of chronically elevated BP in animal models. It was found that elevated BP was associated with atrial fibrosis, significant conduction abnormalities, shortening of atrial wavelength and increased $\mathrm{AF} .{ }^{19}$ In both human and animal models, obstructive sleep apnea has been shown to result in increased atrial fibrosis and increased incidence of extra-pulmonary vein triggers. ${ }^{20}$ Other clinical factors have been implicated in atrial remodeling and consequent $\mathrm{AF}$, including: increased age, obesity, diabetes and heart failure.

\section{Current Status of Management of AF}

Current guidelines suggest that AF treatment should focus on strategies to prevent stroke, and to manage and control heart rate and rhythm. ${ }^{21,22} \mathrm{AF}$ is known to be a chronic disease. Rarely, patients will have a single episode of AF, but the majority of patients have progressively more episodes, or present with persistent AF. As with all chronic diseases, it cannot be cured but can be controlled with effective treatments, as recommended by the guidelines. Our current health care system was designed to address acute illness, rather than chronic disease. AF often occurs in the setting of other diseases, increasing the complexity in determining appropriate therapies. Lone atrial fibrillation occurs in a very small proportion of patients who present with new onset AF and may not even be a real entity. Most often, AF occurs in the setting of other cardiovascular disease, obesity, diabetes, sleep apnea or a combination of the above. In order to effectively manage AF, a 'holistic' approach is necessary. Appropriate management of hypertension, sleep apnea, obesity etc needs to become part of the mainstay of therapy for AF. The CCS AF guidelines recommendations state: "Underlying causes or precipitating factors for AF including hypertension should be identified and treated." ${ }^{23}$ Possible targets for these are included in Table $1 .^{24}$

\section{Lifestyle Modification in AF}

There are several facets of lifestyle modification that can be addressed to modify AF and its resultant outcomes. Risk factor modification has been termed the fourth pillar of AF care, with the first three being rate, rhythm and stroke prevention. The presence of risk factors that are known to promote AF are increasing in prevalence in the population: it is likely that the AF epidemic is greater than predicted. The Framingham study evaluated the incidence and prevalence of $\mathrm{AF}$ and its risk factors over 50 years. 25 In 9511 patients over 50 years of observation, the incidence of AF increased in both men (3.7 vs 13.4 per 1000 person-years) and women ( 2.5 vs 8.5 per 1000 person-years).

Table 1. Potentially Modifiable Risk Markers/Conditions Associated with $\mathrm{AF}^{24}$

\begin{tabular}{|l|l|l|}
\hline Conventional Risk Factors & Emerging Risk Factors & Less Established Risk Factors \\
\hline Advancing age & Subclinical atherosclerosis & Chronic obstructive pulmonary disease \\
\hline male & Borderline hypertension & Left atrial dilatation \\
\hline Coronary artery disease & Chronic kidney disease & Atrial conduction delay/PR interval \\
\hline hypertension & Subclinical hyperthyroidism & Left ventricular diastolic dysfunction \\
\hline Heart failure & Inflammation & Left ventricular hypertrophy/diastolic dysfunction \\
\hline Valvular disease & Elevated natriuretic peptides & Obesity \\
\hline diabetes & Widened pulse pressure & Genetic factors \\
\hline Thyroid disease & Excessive endurance exercise & \\
\hline Obstructive sleep apnea syndrome & Excessive alcohol intake & \\
& Increased height & \\
& Increased birth weight & \\
\cline { 2 - 2 } & Smoking & \\
\hline & Caffeine intake & \\
\hline & Ethnicity & \\
\hline
\end{tabular}


The population attributable risk over time for $\mathrm{AF}$ increased due to higher body mass index (BMI) and diabetes. In a further analysis of the Framingham study, the risk profile of patients as it related to the lifetime risk of atrial fibrillation as assessed. ${ }^{26}$ In this study $4.6 \%$ of patients had an optimal risk profile, defined as no smoking, alcohol consumption within recommended limits, normal body mass index, normotensive, no diabetes, heart failure or myocardial infarction. The lifetime risk of AF was $23.4 \%$ with an optimal risk profile, $33.4 \%$ with a borderline risk profile and $38.4 \%$ with an elevated risk profile (Figure 1).

The diagram in Figure 2 demonstrates the inter-relationship of these facets.

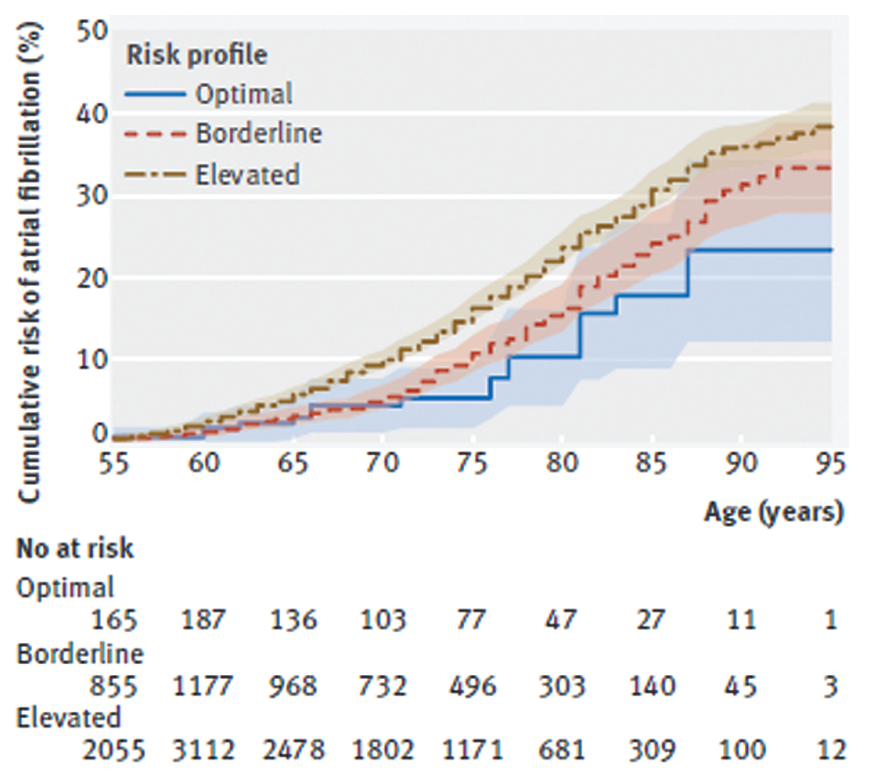

Figure 1. Cumulative risk (\%) for development of $\mathrm{AF}$ according to risk-factor burden: optimal, borderline or elevated. Shading $=95 \%$ confidence intervals. ${ }^{26}$

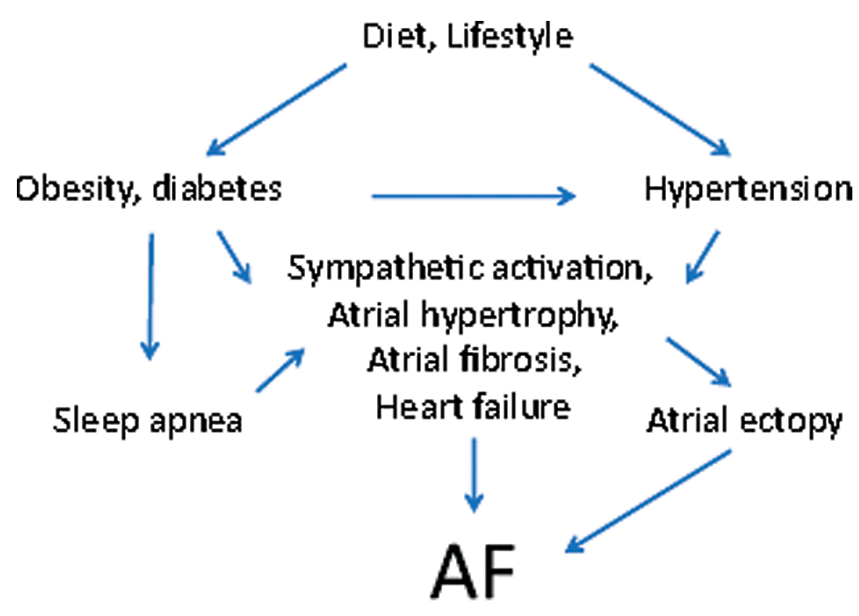

Figure 2. Relationship of cardiovascular risk factors to pathogenesis of AF. Adapted from Van Wagoner et al. ${ }^{27}$

\section{Exercise in AF}

Exercise and physical activity have been shown to improve outcomes in patients with cardiac health conditions such as ischemic heart disease, myocardial infarction and congestive heart failure but its effects on AF remain somewhat unclear. ${ }^{28}$ Exercise has numerous cardiac effects including reducing heart rate and increasing stroke volume. Chronic exercise can further help protect the myocardium from acute ischemia and improve function in response to cardiac insults such as hypertension, myocardial infarction and general wear and tear associated with aging. ${ }^{29}$

The effects of exercise training on health benefits in AF have been evaluated in two systematic reviews. These studies have demonstrated that exercise can increase exercise capacity, improve quality of life, and provide a mechanism of rate control in AF patients. ${ }^{30,31}$ An increase in exercise capacity in AF patients who exercised was demonstrated with increased distances in six-minute walk tests, and increased muscular strength, power and work. Exercise has been shown in some studies to lower resting heart rate by 7 beats per minute and improve heart rate reserve by 15 beats per minute. It should be noted that there are many important parameters to consider when evaluating health outcomes with exercise in AF patients. More work is needed to establish appropriate values for these parameters to maximize beneficial health outcomes.

While many studies have enrolled AF patients in different exercise regimens, only a handful to our knowledge have reported adverse events. ${ }^{31}$ An estimated life-threatening adverse event rate of 1 per 209752 minutes of exercise has been calculated in AF patients. Similarly for non-life-threatening adverse events (e.g., ischemic chest pain, exercise-induced AF), the adverse event rate is estimated to increase to 1 per 9756 minutes of exercise. These studies suggest that exercise is a safe therapy that can be further investigated in AF patients. The significance of this work is highlighted by the fact that patients with AF often present with symptoms of dyspnea, fatigue, palpitations, and decreased exercise capacity. These symptoms not only make it more difficult for patients to exercise, but also reduces their quality of life. Exercise intolerance and sedentary behaviour specifically can drive weight gain and lead to a variety of health issues. The effects of physical activity, as well as physical inactivity ie sedentary behaviour, is unknown in this population. The FIT project examined 64561 adults without AF from 1991 to 2009 and found that one higher metabolic equivalent during treadmill testing was associated with a 7\% lower risk of incident $\mathrm{AF}$, with a stronger relationship amongst obese patients. ${ }^{32}$ The CARDIO FIT study found an association between a gain of $\geq 2$ METS pre and post a tailored exercise program to be associated with improvements in AF burden and symptom severity in a single center cohort study. Malmo et al. performed a single center randomized trial in 50 patients demonstrating a positive effect of intense aerobic interval training to reduce 
recurrent $\mathrm{AF}$ in a population of patients with nonpermanent $\mathrm{AF}^{33}$ Although none of these studies provide definitive evidence regarding the benefits of exercise in AF, they do demonstrate that it is reasonable to have patients with AF perform moderate exercise, without the danger of deleterious effects.

\section{Obesity and AF}

Two randomized trials have shown reduction of AF and improved quality of life in patients with obesity and in patients with heart failure. ${ }^{34,35}$ Abed et al. examined the effect of weight reduction and cardiometabolic risk factor treatment in patients with AF.34 Abed et al. examined the effect of weight reduction and cardiometabolic risk factor treatment in patients with $\mathrm{AF}$ and found an improvement in AF-related quality of life in the experimental arm. The LEGACY study was a cohort study that demonstrated weight reduction of $\geq 10 \%$ to be associated with a six-fold greater probability of arrhythmia-free survival compared with the those who loss less weight or did not lose weight at all. ${ }^{36}$

\section{Blood Pressure and AF}

Hypertension is the most prevalent, independent and potentially modifiable risk for atrial fibrillation..$^{37,38}$ The Framingham study revealed that an electrocardiographic diagnosis of LVH increases the risk of AF by more than 3 fold. ${ }^{39}$ These changes may be a direct result of increased left atrial stretch resulting from increased pressures on the left side of the heart.

Recent studies have found a direct relationship between the risk of $\mathrm{AF}$ and systolic and diastolic BP. Conen et al. evaluated the risk of incident $\mathrm{AF}$ in healthy, middle-aged women as it pertains to $\mathrm{BP}{ }^{40}$ Over a followup period of 12.4 years, the longer term risk of AF increased as the systolic and diastolic BP increased. Only a systolic $\mathrm{BP}<120 \mathrm{mmHg}$ was not associated with an increased HR of developing AF. The primary prevention of AF with antihypertensive therapy has been reported previously. A post-hoc analysis of the Losartan Intervention for Endpoint reduction (LIFE) study found that losartan reduced the incidence of new-onset AF from 10.1 to 6.8 per 1000 patient-years, as compared to atenolol alone; a meta-analysis examining the effect of angiotensin converter enzyme inhibition or angiotensin II receptor blockade on primary prevention of AF in patients with structural heart disease showing similar findings, providing support for the pleotropic effects of these medications. ${ }^{41,42}$

Whether aggressive BP control is of benefit in prevention of AF once it develops remains elusive. Secondary prevention of AF, has not been found to be of benefit using angiotensin converter enzyme inhibition or angiotensin II receptor blockade. The Gruppo Italiano per lo Studio della Sopravvivenza nell'Insufficienza cardiac Atrial Fibrillation (GISSI-AF) study failed to show any benefit in preventing recurrent $\mathrm{AF}$ in a population of $\mathrm{AF}$ with paroxysmal or persistent $\mathrm{AF}^{43}$ The Atrial Fibrillation Clopidogrel Trial with
Irbesartan to Prevent Vascular Events (ACTIVE I) trial also failed to show that the pleotropic effects of angiotensin II receptor blockade had any effect on cardiovascular outcomes in patients with $\mathrm{AF}^{44}$

Parkash et al performed a multicenter, randomized trial examining the effect of an aggressive blood pressure control strategy on recurrent AF after catheter ablation. ${ }^{45}$ This study did not find a benefit to reduction in AF with targeting this risk factor in isolation. The study did however demonstrate that patients whose baseline blood pressure was $<140 \mathrm{~mm} \mathrm{Hg}$, had a significantly lower recurrence of AF after ablation, as compared to those whose blood pressure was elevated at baseline (45\% recurrence for $\mathrm{SBP}<140$ compared to $86 \%$ recurrence for $\mathrm{SBP}>150$, $\mathrm{p}=0.03$ ). Patients older than the age of 61 had a significant benefit to aggressive BP lowering, as compared to younger patients ( $\mathrm{p}$ for interaction $=0.011$ ).

These studies did not find direct benefits to aggressive BP lowering in AF; it remains important, however, to treat this risk factor using the current recommendations from the Canadian Hypertension Education Program guidelines as the target for BP control in AF patients. ${ }^{46}$

\section{Sleep Apnea and AF}

Sleep apnea is a disorder of breathing resulting in hypoventilation, hypopneas and apnea during sleep. It is associated with deleterious outcome when left untreated, particularly from a cardiovascular viewpoint. ${ }^{47}$ It is known to affect $4 \%$ of middle-aged men and $2 \%$ of middle-aged women, as a whole. In the setting of concomitant AF, sleep apnea has been found to occur in up to 1 in 5 patients in the ORBIT AF registry. ${ }^{48}$ The ORBIT registry also demonstrated in reduction in progression to AF, with no effect on mortality or cardiovascular hospitalization. Obstructive sleep apnea and $\mathrm{AF}$ are known to coexist; the risk of AF recurrence is higher in patients who have undergone catheter ablation in patients with obstructive sleep apnea, compared to those patients who do not have it. ${ }^{49}$ Systematic reviews have pooled data from cohort studies that support the beneficial effect of sleep apnea treatment on AF, in combination with catheter ablation of $\mathrm{AF}^{49}$ In a recent clinical trial, there was no benefit of continuous positive airway pressure therapy on cardiovascular outcomes ${ }^{50}$; the effect of CPAP in AF has been shown to be of benefit in cohort studies but no randomized studies have been performed.

\section{Multifactorial Risk Factor Management}

There have been a few studies that have examined the effect of multifactorial risk factor management in patients with AF. The concept here is that treating a single risk factor in isolation may be ineffective, that many patients have multiple risk factors that coexist and overlap to result in further maintenance of AF. Rienstra et al. examined the optimization of heart failure medications, cardiac rehabilitation and aggressive blood pressure control in patients 
with $\mathrm{AF}$ and heart failure, and demonstrated maintenance of sinus rhythm as measured on a 7 day Holter at one year pf $75 \%$ in the intervention group, as compared to $63 \%$ in the control group (OR 1.77, $\mathrm{p}=0.042) .{ }^{35}$ Another single center study examined the role of risk factor management concomitantly with catheter ablation for AF. Pathak et al. performed a single center, cohort study that demonstrated that patients who chose to undergo aggressive risk factor modification had a reduction in AF compared to those who did not with an odds ratio of 4.8 (95\% confidence interval 2.04-11.4)..$^{51}$

\section{Delivery of Lifestyle Modification: Use of Specialized Clinics}

How best to deliver lifestyle modification to this population remains an unanswered question. The use of cardiac rehabilitation programs has been traditionally reserved for those with acute coronary syndromes. Expansion of these programs to include AF patients could result in an overload of this resource, required for a different cardiac population. In addition, ensuring delivery of care across geographic boundaries, particularly in Canada is important. Using 'bricks and mortar' programs, this becomes difficult. Specialized AF clinics have garnered significant attention recently. The studies that have been performed are discussed below.

Gillis et al. published their preliminary data from this type of clinic, which showed encouraging results. ${ }^{52}$ The clinic accepted 20-40 patients per week for assessment. The time for specialist assessment was brought to $38+/-31$ days, as compared to $221+/$ 774 days in the year prior to the formation of the clinic. The number of emergency department visits and hospitalizations were dramatically reduced, $82 \%$ and $56 \%$ respectively. Gillis et al. first established their clinic through an innovative fund from their health care region. The results were so positive that funds to permanently establish this clinic were allocated. The Integrated Management to AF study (ICAT-AF) demonstrated that a nurseled, physician supervised program providing education and management of risk factors (before-after study; $n=433$ ) could improve guideline adherence, and improve health outcomes (decreased AF-related ED visits and hospitalizations by $29 \%$ in new onset $\mathrm{AF}^{53}$ The largest study examining this model of care was performed in the Netherlands. Hendriks et al. demonstrated a reduction in $\mathrm{CV}$ mortality in a randomized controlled trial of a nurse-led, physician supervised AF clinic in the Netherlands, as compared to usual care. ${ }^{54}$ Cardiovascular death occurred at a rate of $1.1 \%$ in the nurse-led care vs. $3.9 \%$ in the usual care group (hazard ratio: 0.28 ; 95\% CI: 0.09-0.85; $\mathrm{P}=0.025$ ); cardiovascular hospitalization was reduced to 13.5 from $19.1 \%$ in the usual care group (hazard ratio: 0.66 ; $95 \% \mathrm{CI}: 0.46-0.96, \mathrm{P}=0.029$ ). The Hendriks study utilized a computer-based algorithm to assist the nurse with the AF care. This computer-based algorithm is costly to apply widely and as such the outcomes observed by the study may not be generalizable.
Multiple prior studies have demonstrated the effectiveness of multidisciplinary care in peri-procedural management. Recent reviews have emphasized the importance of integrated care for heart rhythm disorders. Greater coordination of care with nursing interventions has demonstrated more efficient use of resources, ${ }^{55}$ and better coordination of heart rhythm procedures, ${ }^{56,57}$ as well as better implementation of other cardiac therapies. ${ }^{58,59}$

In these studies, the observed reduction in primary outcome events may be attributable to several factors including consistency of patient education delivered by a nurse, repeated encounters, improved guideline adherence, particularly in OAC use, as well as risk factor management, resulting in the observed reduction in cardiovascular events. Significant delays in receiving guidelineindicated therapies, specifically appropriate anticoagulation, could lead to adverse cardiovascular outcomes. Based on previously published data, the monthly incidence of stroke with non-valvular AF ranges from $0.23 \%$ to $1.5 \%$, depending on CHADS2 score. ${ }^{60}$ Given wait times for specialist assessment, whether through a specialized AF clinic or specialist usual care, the incidence of stroke could be reduced by improving anticoagulation at the time of AF diagnosis.

\section{Conclusion}

The difficulty arises in how patients with AF and its associated risk factors should be managed. The use of specialized clinics may result in patients receiving care from various specialists, that may lead to multiple and potentially conflicting treatment recommendations. As the complexity of medical problems plaguing an individual patient increases, so does the number of specialty clinics that he/she may be exposed to. This results in greater need for centralization of a patient's care with their family physician. Risk factor management may be improved by a form of integrated care. Using a combination of pharmacologic and non-pharmacologic treatments (ie focus on lifestyle), risk factors that aggravate and trigger AF likely need to become a part of our usual armamentarium of care in AF patients. The most expeditious model of care for AF remains a challenge in our resource-constrained environment, but remains an important focus to improve outcomes associated with AF.

\section{References}

1. Lip GY, Tse HF. Management of atrial fibrillation. Lancet 2007;370:604-618.

2. Wolf PA, Dawber TR, Thomas HE, Jr., Kannel WB. Epidemiologic assessment of chronic atrial fibrillation and risk of stroke: the Framingham study. Neurology 1978;28:973-977.

3. Wolf PA, Abbott RD, Kannel WB. Atrial fibrillation: a major contributor to stroke in the elderly. The Framingham Study. ArchInternMed 1987;147:1561-1564.

4. Benjamin EJ, Wolf PA, D’Agostino RB, Silbershatz H, Kannel WB, Levy D. Impact of atrial fibrillation on the risk of death: the Framingham Heart Study. Circulation 1998;98:946-52.

5. Stroke. Heart and Stroke Foundation of Canada 2013. 
6. Parkash R, Maisel WH, Toca FM, Stevenson WG. Atrial fibrillation in heart failure: high mortality risk even if ventricular function is preserved. AmHeart J 2005;150:701-706.

7. Kirchhof P, Schmalowsky J, Pittrow D et al. Management of patients with atrial fibrillation by primary-care physicians in Germany: 1-year results of the ATRIUM registry. Clin Cardiol 2014;37:277-84.

8. Wolf PA, Mitchell JB, Baker CS, Kannel WB, D'Agostino RB. Impact of atrial fibrillation on mortality, stroke, and medical costs. Arch Intern Med 1998;158:229-34.

9. McDonald AJ, Pelletier AJ, Ellinor PT, Camargo CA, Jr. Increasing US emergency department visit rates and subsequent hospital admissions for atrial fibrillation from 1993 to 2004. AnnEmergMed 2008;51:58-65.

10. Khaykin Y, Morillo CA, Skanes AC, McCracken A, Humphries K, Kerr CR. Cost comparison of catheter ablation and medical therapy in atrial fibrillation. Journal of cardiovascular electrophysiology 2007;18:907-13.

11. Gladstone DJ, Bui E, Fang J et al. Potentially preventable strokes in high-risk patients with atrial fibrillation who are not adequately anticoagulated. Stroke 2009;40:235-240.

12. Ogilvie IM, Newton N, Welner SA, Cowell W, Lip GY. Underuse of oral anticoagulants in atrial fibrillation: a systematic review. Am J Med 2010;123:638-645.

13. Nattel S. New ideas about atrial fibrillation 50 years on. Nature 2002;415:219-226.

14. Haissaguerre M, Jais P, Shah DC et al. Spontaneous initiation of atrial fibrillation by ectopic beats originating in the pulmonary veins. N Engl J Med 1998;339:659-666.

15. Dixit S, Marchlinski FE, Lin D et al. Randomized ablation strategies for the treatment of persistent atrial fibrillation: RASTA study. Circ Arrhythm Electrophysiol 2012;5:287-94.

16. Saygili E, Rana OR, Saygili E et al. Losartan prevents stretch-induced electrical remodeling in cultured atrial neonatal myocytes. Am J Physiol Heart Circ Physiol 2007;292:H2898-H2905.

17. Wijffels MC, Kirchhof CJ, Dorland R, Allessie MA. Atrial fibrillation begets atrial fibrillation. A study in awake chronically instrumented goats. Circulation 1995;92:1954-1968.

18. Scherlag BJ, Patterson E, Po SS. The neural basis of atrial fibrillation. J Electrocardiol 2006.

19. Kistler PM, Sanders P, Dodic M et al. Atrial electrical and structural abnormalities in an ovine model of chronic blood pressure elevation after prenatal corticosteroid exposure: implications for development of atrial fibrillation. Eur Heart J 2006;27:3045-3056.

20. Anter E, Di Biase L, Contreras-Valdes FM et al. Atrial Substrate and Triggers of Paroxysmal Atrial Fibrillation in Patients With Obstructive Sleep Apnea. Circ Arrhythm Electrophysiol 2017;10.

21. Gillis AM, Verma A, Talajic M, Nattel S, Dorian P. Canadian cardiovascular society atrial fibrillation guidelines 2010: rate and rhythm management. CanJCardiol 2011;27:47-59.

22. Cairns JA, Connolly S, McMurtry S, Stephenson M, Talajic M. Canadian cardiovascular society atrial fibrillation guidelines 2010: prevention of stroke and systemic thromboembolism in atrial fibrillation and flutter. CanJCardiol 2011;27:74-90.

23. Healey JS, Parkash R, Pollak PT, Tsang TS, Dorian P. Canadian Cardiovascular Society 2010 Atrial Fibrillation Guidelines: Chapter 2 - Atrial Fibrillation: Etiology and Initial Investigations. CanJCardiol 2011.

24. Wyse DG, Van Gelder IC, Ellinor PT et al. Lone atrial fibrillation: does it exist? J Am Coll Cardiol 2014;63:1715-23.

25. Schnabel RB, Yin X, Gona P et al. 50 year trends in atrial fibrillation prevalence, incidence, risk factors, and mortality in the Framingham Heart Study: a cohort study. Lancet 2015;386:154-62.

26. Staerk L, Wang B, Preis SR et al. Lifetime risk of atrial fibrillation according to optimal, borderline, or elevated levels of risk factors: cohort study based on longitudinal data from the Framingham Heart Study. BMJ 2018;361:k1453.

27. Van Wagoner DR, Piccini JP, Albert CM et al. Progress toward the prevention and treatment of atrial fibrillation: A summary of the Heart Rhythm Society Research Forum on the Treatment and Prevention of Atrial Fibrillation, Washington, DC, December 9-10, 2013. Heart Rhythm 2015;12:e5-e29.
28. Belardinelli R, Georgiou D, Ginzton L, Cianci G, Purcaro A. Effects of moderate exercise training on thallium uptake and contractile response to low-dose dobutamine of dysfunctional myocardium in patients with ischemic cardiomyopathy. Circulation 1998;97:553-561.

29. Osbak PS, Mourier M, Kjaer A, Henriksen JH, Kofoed KF, Jensen GB. A randomized study of the effects of exercise training on patients with atrial fibrillation. AmHeart J 2011;162:1080-1087.

30. Reed JL, Mark AE, Reid RD, Pipe AL. The effects of chronic exercise training in individuals with permanent atrial fibrillation: a systematic review. CanJCardiol 2013;29:1721-1728.

31. Giacomantonio NB, Bredin SS, Foulds HJ, Warburton DE. A systematic review of the health benefits of exercise rehabilitation in persons living with atrial fibrillation. CanJCardiol 2013;29:483-491.

32. Qureshi WT, Alirhayim Z, Blaha MJ et al. Cardiorespiratory Fitness and Risk of Incident Atrial Fibrillation: Results From the Henry Ford Exercise Testing (FIT) Project. Circulation 2015;131:1827-34.

33. Pathak RK, Elliott A, Middeldorp ME et al. Impact of CARDIOrespiratory FITness on Arrhythmia Recurrence in Obese Individuals With Atrial Fibrillation: The CARDIO-FIT Study. J Am Coll Cardiol 2015;66:985-96.

34. Abed HS, Wittert GA, Leong DP et al. Effect of weight reduction and cardiometabolic risk factor management on symptom burden and severity in patients with atrial fibrillation: a randomized clinical trial. JAMA 2013;310:2050-2060.

35. Rienstra M, Hobbelt AH, Alings M et al. Targeted therapy of underlying conditions improves sinus rhythm maintenance in patients with persistent atrial fibrillation: results of the RACE 3 trial. Eur Heart J 2018.

36. Pathak RK, Middeldorp ME, Meredith M et al. Long-Term Effect of Goal Directed Weight Management in an Atrial Fibrillation Cohort: A Long-term Follow-Up StudY (LEGACY Study). J Am Coll Cardiol 2015.

37. Parkash R, Verma A, Tang AS. Persistent atrial fibrillation: current approach and controversies. Curr Opin Cardiol 2010;25:1-7.

38. Healey JS, Connolly SJ. Atrial fibrillation: hypertension as a causative agent, risk factor for complications, and potential therapeutic target. Am J Cardiol 2003;91:9G-14G.

39. Kannel WB, Wolf PA, Benjamin EJ, Levy D. Prevalence, incidence, prognosis, and predisposing conditions for atrial fibrillation: population-based estimates. Am J Cardiol 1998;82:2N-9N.

40. Conen D, Tedrow UB, Koplan BA, Glynn RJ, Buring JE, Albert CM. Influence of systolic and diastolic blood pressure on the risk of incident atrial fibrillation in women. Circulation 2009;119:2146-2152.

41. Wachtell K, Lehto M, Gerdts E et al. Angiotensin II receptor blockade reduces new-onset atrial fibrillation and subsequent stroke compared to atenolol: the Losartan Intervention For End Point Reduction in Hypertension (LIFE) study. J Am Coll Cardiol 2005;45:712-719.

42. Healey JS, Baranchuk A, Crystal E et al. Prevention of atrial fibrillation with angiotensin-converting enzyme inhibitors and angiotensin receptor blockers: a meta-analysis. J Am Coll Cardiol 2005;45:1832-1839.

43. Disertori M, Latini R, Barlera S et al. Valsartan for prevention of recurrent atrial fibrillation. N Engl J Med 2009;360:1606-1617.

44. Yusuf S, Healey JS, Pogue J et al. Irbesartan in patients with atrial fibrillation. N Engl J Med 2011;364:928-38.

45. Parkash R, Wells GA, Sapp JL et al. Effect of Aggressive Blood Pressure Control on the Recurrence of Atrial Fibrillation After Catheter Ablation: A Randomized, Open-Label Clinical Trial (SMAC-AF [Substrate Modification With Aggressive Blood Pressure Control]). Circulation 2017;135:1788-1798.

46. Leung AA, Daskalopoulou SS, Dasgupta K et al. Hypertension Canada's 2017 Guidelines for Diagnosis, Risk Assessment, Prevention, and Treatment of Hypertension in Adults. Can J Cardiol 2017;33:557-576.

47. Marini C, De SF, Sacco S et al. Contribution of atrial fibrillation to incidence and outcome of ischemic stroke: results from a population-based study. Stroke 2005;36:1115-1119.

48. Holmqvist F, Guan N, Zhu Z et al. Impact of obstructive sleep apnea and continuous positive airway pressure therapy on outcomes in patients with atrial fibrillation-Results from the Outcomes Registry for Better Informed 
Treatment of Atrial Fibrillation (ORBIT-AF). American heart journal 2015;169:647-654 e2.

49. Li L, Wang ZW, Li J et al. Efficacy of catheter ablation of atrial fibrillation in patients with obstructive sleep apnoea with and without continuous positive airway pressure treatment: a meta-analysis of observational studies. Europace 2014;16:1309-14.

50. McEvoy RD, Antic NA, Heeley E et al. CPAP for Prevention of Cardiovascular Events in Obstructive Sleep Apnea. N Engl J Med 2016;375:919-31.

51. Pathak RK, Middeldorp ME, Lau DH et al. Aggressive risk factor reduction study for atrial fibrillation and implications for the outcome of ablation: the ARREST-AF cohort study. J Am Coll Cardiol 2014;64:2222-2231.

52. Gillis AM, Burland L, Arnburg B et al. Treating the right patient at the right time: an innovative approach to the management of atrial fibrillation. Can J Cardiol 2008;24:195-198.

53. Carter L, Gardner M, Magee K et al. An Integrated Management Approach to Atrial Fibrillation. J AmHeart Assoc 2016;5.

54. Hendriks JM, de WR, Crijns HJ et al. Nurse-led care vs. usual care for patients with atrial fibrillation: results of a randomized trial of integrated chronic care vs. routine clinical care in ambulatory patients with atrial fibrillation. Eur Heart J 2012;33:2692-2699.
55. Gross PA, Patriaco D, McGuire K, Skurnick J, Teichholz LE. A nurse practitioner intervention model to maximize efficient use of telemetry resources. Jt Comm J Qual Improv 2002;28:566-573.

56. Shelton RJ, Allinson A, Johnson T, Smales C, Kaye GC. Four years experience of a nurse-led elective cardioversion service within a district general hospital setting. Europace 2006;8:81-85.

57. Boodhoo L, Bordoli G, Mitchell AR, Lloyd G, Sulke N, Patel N. The safety and effectiveness of a nurse led cardioversion service under sedation. Heart 2004;90:1443-1446.

58. Andersen MK, Markenvard JD, Schjott H, Nielsen HL, Gustafsson F. Effects of a nurse-based heart failure clinic on drug utilization and admissions in a community hospital setting. Scand Cardiovasc J 2005;39:199-205.

59. Andersson B, Kjork E, Brunlof G. Temporal improvement in heart failure survival related to the use of a nurse-directed clinic and recommended pharmacological treatment. Int J Cardiol 2005;104:257-263.

60. Olesen JB, Lip GY, Hansen ML et al. Validation of risk stratification schemes for predicting stroke and thromboembolism in patients with atrial fibrillation: nationwide cohort study. BMJ 2011;342:d124. 